\section{Drs. Moon and Min reply}

\section{To the Editor:}

We highly appreciate the interest ${ }^{1}$ in our paper ${ }^{2}$ suggesting the potential role of cytokine interleukin 34 (IL-34) in rheumatoid arthritis (RA) pathogenesis. Besides our study, there have been other studies suggesting the possible pathophysiological role of IL-34 in human RA ${ }^{3,4,5}$. While there are conflicting results, it could be speculated that IL-34 can contribute to the structural joint damage and/or systemic osteoporosis in inflammatory arthritis, including RA and ankylosing spondylitis ${ }^{4}$. In our study, there was no significant association between the total Sharp score (TSS) and the serum level of IL-34. However, a study by Chang, et $a l^{4}$ showed that baseline IL-34 levels were positively correlated to $\triangle$ SHS (modified Sharp/van der Heijde score)/year and also rheumatoid factor (RF) in patients with RA, suggesting the association between rapid radiographic progression and high circulating IL-34 levels. It should be noted that the radiographic damage score used in our study (TSS) was different from $\Delta$ SHS. More interestingly, our study demonstrated that the synovial fluid (SF) level of IL-34 was positively correlated with the SF receptor activator of nuclear factor- $\mathrm{\kappa B}$ concentration, indicating the potential role of IL-34 in increased osteoclastogenesis in RA. Basically, both IL-34 and colony-stimulating factor 1 (CSF-1) are common ligands for the CSF-1 receptor, highly suggesting a pro-osteoclastogenic role of IL-34. Although further studies are needed to determine whether IL-34 occupies a dominant role in RA pathogenesis rather than CSF-1, previous studies demonstrating the pro-osteoclastogenic role of IL-34 and its response to proinflammatory cytokines such as tumor necrosis factor- $\alpha^{6,7}$ suggested that IL-34 can be a potential treatment target in human RA, especially in the aspect of joint destruction. IL-34 concentrations were higher in seropositive RA ${ }^{3}$. That finding is consistent with our study. We also found that serum IL-34 levels were positively correlated with RF and anticyclic citrullinated peptide antibody titers ${ }^{2}$.

Taken together, previous studies including our paper have suggested the possible pathophysiological roles of IL-34 in RA. However, further studies are needed to reveal more roles of IL-34 in RA pathogenesis, including in terms of autoantibody production.
SU-JIN MOON, MD, PhD; JUN-KI MIN, MD, PhD, Division of Rheumatology, Department of Internal Medicine, College of Medicine, The Catholic University of Korea, Bucheon St. Mary's Hospital, Bucheon, South Korea. Address correspondence to

Dr. J-K. Min, Sosa-2, Wonmi-gu, Bucheon, Republic of Korea.

E-mail:min6403@catholic.ac.kr

\section{REFERENCES}

1. He W, Xu X-D, Dong Y-M, Wu H. Interleukin-34: a potential diagnostic and therapeutic target for rheumatoid arthritis. J Rheumatol 2015;42:553.

2. Moon SJ, Hong YS, Ju JH, Kwok SK, Park SH, Min JK. Increased levels of interleukin 34 in serum and synovial fluid are associated with rheumatoid factor and anticyclic citrullinated peptide antibody titers in patients with rheumatoid arthritis. J Rheumatol 2013;40:1842-9.

3. Tian Y, Shen H, Xia L, Lu J. Elevated serum and synovial fluid levels of interleukin-34 in rheumatoid arthritis: possible association with disease progression via interleukin-17 production. J Interferon Cytokine Res 2013;33:398-401.

4. Chang SH, Choi BY, Choi J, Yoo JJ, Ha YJ, Cho HJ, et al. Baseline serum interleukin-34 levels independently predict radiographic progression in patients with rheumatoid arthritis. Rheumatol Int 2014 Jun 11 (E-pub ahead of print).

5. Hwang SJ, Choi B, Kang SS, Chang JH, Kim YG, Chung YH, et al. Interleukin-34 produced by human fibroblast-like synovial cells in rheumatoid arthritis supports osteoclastogenesis. Arthritis Res Ther 2012;14:R14.

6. Chen Z, Buki K, Vääräniemi J, Gu G, Väänänen HK. The critical role of IL-34 in osteoclastogenesis. PLoS One 2011;6:e18689.

7. Boström EA, Lundberg P. The newly discovered cytokine IL-34 is expressed in gingival fibroblasts, shows enhanced expression by pro-inflammatory cytokines, and stimulates osteoclast differentiation. PloS One 2013;8:e81665.

J Rheumatol 2015;42:3; doi:10.3899/jrheum.141405 